\title{
A novel risk stratification for predicting prognosis of colorectal cancer patients with bone metastasis
}

\author{
Xiaolong Ma ${ }^{\#}$, Xu Guan ${ }^{\#}$, Chenxi Ma1, Jichuan Quan'1, Zhixun Zhao', Haipeng Chen', Haiyang Huang', \\ Ran Wei ${ }^{1}$, Zheng Liu' ${ }^{1}$, Zheng Jiang', Yinggang Chen ${ }^{2}$, Xishan Wang ${ }^{1}$ \\ ${ }^{1}$ Department of Colorectal Surgery, National Cancer Center/National Clinical Research Center for Cancer/Cancer Hospital, Chinese Academy of Medical \\ Sciences and Peking Union Medical College, Beijing, China; ${ }^{2}$ Department of Colorectal Surgery, National Cancer Center/National Clinical Research \\ Center for Cancer/Cancer Hospital \& Shenzhen Hospital, Chinese Academy of Medical Sciences and Peking Union Medical College, Shenzhen, China \\ Contributions: (I) Conception and design: X Ma, X Guan, C Ma; (II) Administrative support: X Wang, Y Chen; (III) Provision of study materials or \\ patients: J Quan, Z Zhao, H Huang, R Wei; (IV) Collection and assembly of data: X Ma, C Ma; (V) Data analysis and interpretation: H Chen, Z Liu, \\ Z Jiang; (VI) Manuscript writing: All authors; (VII) Final approval of manuscript: All authors. \\ \#These authors contributed equally to this work. \\ Correspondence to: Xishan Wang. Department of Colorectal Surgery, National Cancer Center/National Clinical Research Center for Cancer/Cancer \\ Hospital, Chinese Academy of Medical Sciences and Peking Union Medical College, Beijing 100021, China. Email: wxshan_1208@126.com; Yinggang \\ Chen. Department of Colorectal Surgery, National Cancer Center/National Clinical Research Center for Cancer/Cancer Hospital \& Shenzhen \\ Hospital, Chinese Academy of Medical Sciences and Peking Union Medical College, Shenzhen 518116, China. Email: chygang777@163.com.
}

\begin{abstract}
Background: Our understanding in prognosis of bone metastasis (BM) from colorectal cancer (CRC) is limited. We aimed to establish a clinical risk stratification for individually predicting the survival of CRC patients with BM.

Methods: A total of 200 CRC patients with BM were included in this study. Survival time from BM diagnosis was estimated using the Kaplan-Meier method. The multivariable COX regression model identified the risk factors on cancer specific survival (CSS). Based on weighted scoring system, the stratification model was constructed to classify patients with BM according to prognostic risk. Discrimination power and calibration ability of risk stratification were measured.

Results: The median CSS time was 11 months after BM diagnosis. Lymph node metastasis, Carbohydrate antigen 199 (CA199) levels, bone involvement, Karnofsky Performance Status (KPS) scores, primary tumor resection, bisphosphonates therapy and radiotherapy were identified as predictors of CSS. Four risk groups were stratified according to weighted scoring system, including low risk, medium risk, medium-high risk and high risk group, with 35,16, 9 and 5 months of median CSS, respectively $(\mathrm{P}=0.000)$. The risk stratification displayed good accuracy in predicting CSS, with acceptable discrimination and calibration.

Conclusions: This novel risk stratification predicts CSS in CRC patient with BM using easily accessible clinicopathologic factors, which is recommended for use in individualized clinical decision making in patient with BM.
\end{abstract}

Keywords: Colorectal cancer (CRC); bone metastasis (BM); prognostic factors; risk stratification

Submitted Dec 11, 2020. Accepted for publication Mar 28, 2021.

doi: 10.21037/jgo-20-586

View this article at: http://dx.doi.org/10.21037/jgo-20-586

\section{Introduction}

As the most common cancer and the second most common cause of death worldwide, colorectal cancer (CRC) is likely to metastasize to liver, followed by lungs and peritoneal cavity, yet rarely to bone $(1,2)$. It is reported that the incidence of $\mathrm{BM}$ from $\mathrm{CRC}$ is $6.0-10.4 \%$ (3) but has gradually increased in recent years (4). Bone metastasis $(\mathrm{BM})$ is a rare and special type of metastasis, presenting 
with very poor prognosis (5). BM is completely a special distant metastasis compared to liver or lung metastases, and its uniqueness and particularity should be attracted more attention in current clinical practice.

The diversity and complexity of metastasis provide a big challenge in the treatment of CRC with BM. How to make treatment plan and predict the treatment effect is very crucial. Either overtreatment or undertreatment for BM could have an adverse impact on prognosis and quality of life of patients. However, because of low incidence and poor prognosis of $\mathrm{BM}$, the current treatment strategy for this group of patients has relatively been ignored. Previous studies with regard to the treatment plan decision of CRC patients with BM mainly originated from clinical experience and lack standardized guidance, because of the relative rarity of BM in CRC patients.

Previous studies have reported about the characteristics and clinical outcomes of BM from CRC $(5,6)$. However, risk model for predicting survival outcome for CRC patients with $\mathrm{BM}$ are scarce. Construction of a clinically useful risk stratification based on clinicopathologic factors will allow classification of patients according to different prognosis, which have important clinical value in guiding followup care and treatment. Therefore, the aims of our study were to (I) identify the clinicopathological characteristics and prognostic factors of BM from CRC; (II) to develop a comprehensive and practical risk stratification for evaluation of prognosis of CRC patients after BM diagnosis. We present the following article in accordance with the Tripod reporting checklist (available at http://dx.doi.org/10.21037/ jgo-20-586).

\section{Methods}

The study was conducted in accordance with the Declaration of Helsinki (as revised in 2013). This study was approved by the Ethics Committee of Cancer Hospital, Chinese Academy of Medical Sciences (NCC2019S-060) and informed consent was taken from all the patients.

\section{Data resources and study population}

Patients who were diagnosed with BM from CRC between January 2008 and December 2017 at Cancer Hospital, Chinese Academy of Medical Sciences, were retrospectively identified. Patients without follow-up information or patients with $\mathrm{BM}$ from other malignant tumors were excluded from our study. The primary CRC lesion was confirmed by histopathological examination. The American Joint Committee on Cancer (AJCC) TNM stage and $\mathrm{BM}$ were identified by histopathological or imaging examinations such as standard $\mathrm{X}$-rays, whole-body bone scans, computed tomography (CT), magnetic resonance imaging (MRI) and positron emission tomographycomputed tomography (PET-CT). For the number of bone metastases, two adjacent vertebral metastases were classified into the solitary bone involvement, while non-consecutive metastases or more than 2 consecutive vertebral metastases were classified as multiple bone involvement. Synchronous $\mathrm{BM}$ refers to bone metastases found within 3 months after the diagnosis of CRC. Metachronous BM refers to bone metastases found more than 3 months after the diagnosis of CRC. The cancer specific survival (CSS) was defined as the time from the BM diagnosis until cancer-associated death or the end of follow up.

\section{Prognostic factors}

Clinicopathological data and treatment methods were collected from medical records or via telephone followups. The last follow-up time was January 2020. Several variables were analyzed including age ( $<60 v s . \geq 60$ years), sex (female $v s$. male), basic disease (no vs. yes), timing of BM diagnosis (metachronous $v s$. synchronous), primary tumor location (rectum vs. left hemicolon vs. right hemicolon), pathological type of tumor (adenocarcinoma $v s$. mucinous adenocarcinoma $v s$. signet-ring cell carcinoma $v s$. others), tumor grade (I/II vs. III/IV), AJCC T stage (T1/T2 vs. T3/ T4), AJCC N stage (N0 vs. N1/N2), carcinoembryonic antigen (CEA) levels at BM diagnosis (negative vs. positive), carbohydrate antigen 199 (CA199) levels at BM diagnosis (negative $v s$. positive), alkaline phosphatase (ALP) levels at BM diagnosis (negative vs. positive), bone involvement (solitary $v s$. multiple), KPS at BM diagnosis $(\geq 80 v s .<80$ ), extra-osseous metastases (no vs. yes), primary tumor resection (no vs. yes), systemic treatment for BM (chemotherapy alone $v s$. chemotherapy plus targeted therapy), bisphosphonates for BM (no vs. yes), radiotherapy for BM (no vs. yes) and operation for BM (no vs. yes).

\section{Risk stratification}

We developed a weighted scoring system and assigned points for each adverse prognostic factor according to its beta coefficients $(\beta)$ value. The insignificant risk factors $(\mathrm{P}>0.05)$ received 0 point. Adverse factors $(\mathrm{P}<0.05)$ with 
$0<\beta<0.5$ received 1 point; those with $0.5 \leq \beta<1$ received 2 points. Risk groups were stratified based on total risk scores of prognostic factors.

\section{Statistical analysis}

The CSS was assessed with Kaplan-Meier method, with the log-rank tests used to compare subgroups. In order to reduce the impact of sample size, factors with $\mathrm{P}<0.20$ in univariate Kaplan-Meier analyses (7) were finally tested in multivariable COX regression analysis via a backward stepwise selection process. Hazard ratio (HR), corresponding $95 \%$ confidence interval (CI) and $\beta$ were also calculated by COX regression model. The discrimination power of risk stratification was measured by calculating the area under the time-dependent receiver operating characteristic (AUROC) curve. Calibration curves were provided to internally evaluate the calibration ability of risk stratification. All statistical analyses were performed with SPSS version 25.0 for Mac or $\mathrm{R}$ version 3.6.0. It is considered as statistically significant when $\mathrm{P}<0.05$.

\section{Results}

\section{Patients characteristics}

Finally, 200 patients with BM from CRC were enrolled in our study, with 195 deaths (97.5\%). The median age was 58 years (range from 19 to 84 years), and most of patients were male (59.5\%). There was predominance of metachronous BM (68.5\%) and rectal cancer (55.0\%) in cohort. Most of patients were diagnosed with adenocarcinoma $(86.0 \%)$, advanced AJCC T stage $(68.5 \%)$ and $\mathrm{N}$ stage $(71.5 \%)$. Patients with KPS $\geq 80$ points accounted for $75.5 \%$ at the time of BM diagnosis. Table 1 summarizes the clinicopathological features of the patients.

\section{Patterns of BM and extra-osseous metastasis}

In total of 200 patients, there were 108 patients (54.0\%) having BM to solitary site and 92 patients (46.0\%) having $\mathrm{BM}$ to multiple sites respectively (Table 1). The most common metastatic sites were the spine $(\mathrm{n}=141,70.5 \%)$, pelvis $(\mathrm{n}=121,60.5 \%)$, long bones $(\mathrm{n}=53,26.5 \%)$ and ribs $(\mathrm{n}=49,24.5 \%)$, respectively. Only 8 patients $(4.0 \%)$ were diagnosed with skull metastasis.

There were 23 patients (11.5\%) having isolated BM, while the remaining 177 patients (88.5\%) having metastases to other distant organs (Table 1). The most common site of extra-osseous metastases was lung $(\mathrm{n}=104,52 \%)$, followed by liver ( $\mathrm{n}=101,50.5 \%)$, distant lymph nodes $(\mathrm{n}=90,45 \%)$, ovary $(\mathrm{n}=28,14.0 \%)$, adrenal gland $(\mathrm{n}=14,7.0 \%)$, brain $(\mathrm{n}=12,6.0 \%)$ and peritoneum $(\mathrm{n}=10,5.0 \%)$.

\section{Treatments for BM}

The treatments for patients after diagnosis of BM were seen in Table 1. There were 66 patients skipped surgery for primary tumor mainly due to the inability of radical resection. All patients received chemotherapy after diagnosis of BM, and some ( $\mathrm{n}=79,39.5 \%)$ also received targeted therapy. There were 85 patients $(42.5 \%)$ and 57 patients $(28.5 \%)$ receiving bisphosphonates and radiotherapy, respectively. Only 3 patients (1.5\%) took surgery for metastatic tumors of bone because of spinal cord compression.

\section{CSS and prognostic factors}

Median CSS after BM diagnosis was 11 months (95\% CI: 9.7-12.3). The 1-, 2- and 3-year CSS rate after BM diagnosis was $30.0 \%, 17.0 \%$ and $7.0 \%$, respectively.

Potential prognostic variables with $\mathrm{P}<0.20$ in univariate Kaplan-Meier analyses were represented in Figure 1, which were identified for multivariate analysis. The COX multivariate analysis revealed high AJCC N stage (HR: 1.652, 95\% CI: $1.055-2.587, \mathrm{P}=0.028)$, positive CA199 levels (HR: 1.460, 95\% CI: 1.061-2.009, $\mathrm{P}=0.020$ ), multiple bone involvement (HR: 1.534, 95\% CI: 1.117-2.105, $\mathrm{P}=0.008$ ) and $\mathrm{KPS}<80$ at $\mathrm{BM}$ diagnosis (HR: $1.527,95 \%$ CI: 1.071-2.176, $\mathrm{P}=0.019)$ as independent variables related to worse CSS. While primary tumor resection (HR: 0.627, 95\% CI: 0.429-0.916, $\mathrm{P}=0.016$ ), bisphosphonates therapy (HR: $0.581,95 \%$ CI: $0.429-0.788, \mathrm{P}=0.000$ ) and radiotherapy for BM (HR: 0.578, 95\% CI: 0.407-0.822, $\mathrm{P}=0.002)$ were independent variables related to better CSS (Table 2). Kaplan-Meier curves of each significant prognostic factor were shown in Figure 1.

\section{Risk stratification for CSS}

We developed a weighted scoring system according to $\beta$ value of prognostic factors identified from the multivariable analysis. The details were seen in Table 2. Adverse factors including positive CA199 levels, multiple bone involvement and KPS scores $<80$ at BM diagnosis were assigned for 1 point, respectively. Other adverse factors including AJCC N1 or N2 stage, no primary tumor resection, no 
Table 1 Clinicopathological characteristics, treatment and univariate survival analysis

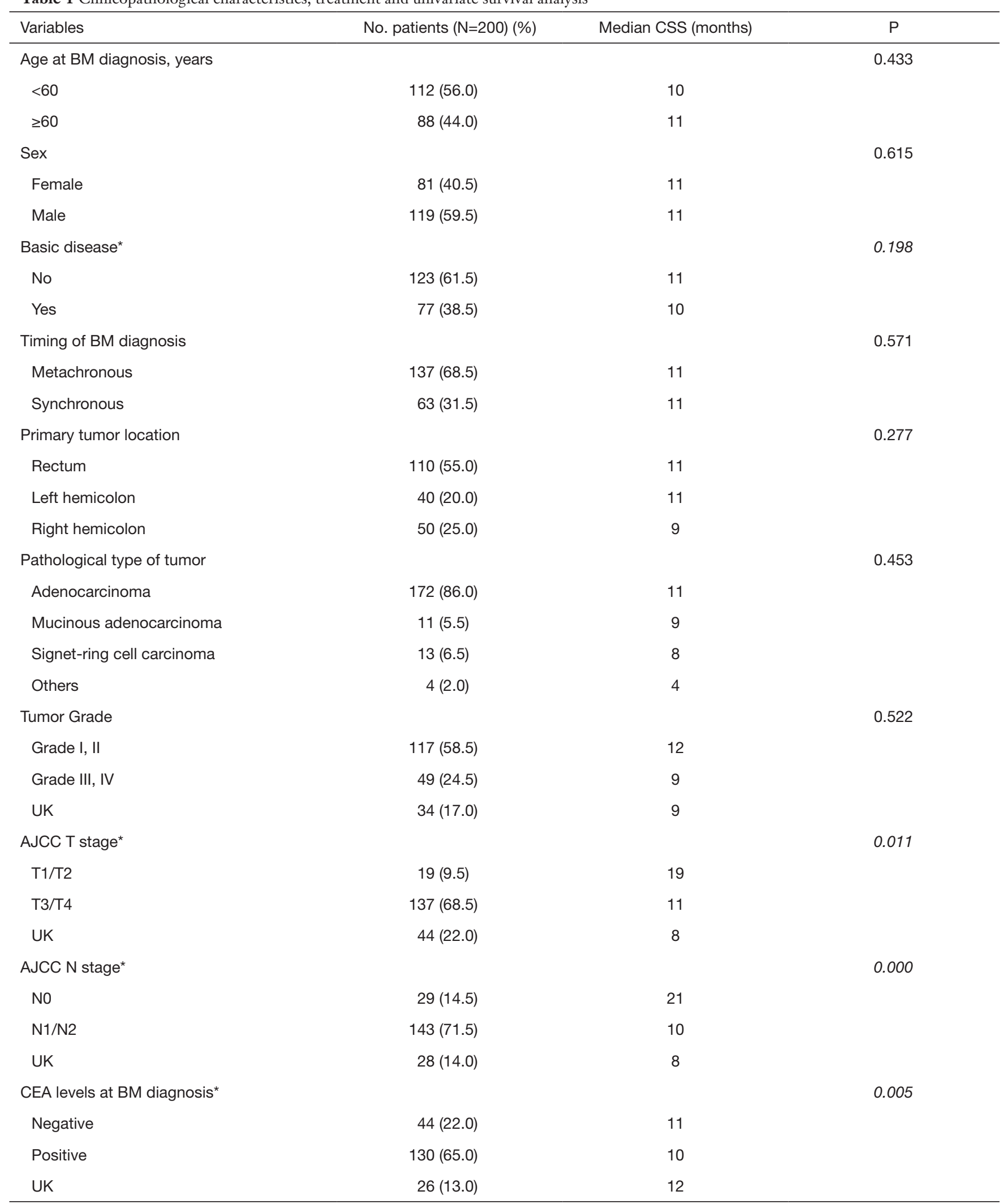

Table 1 (continued) 
Table 1 (continued)

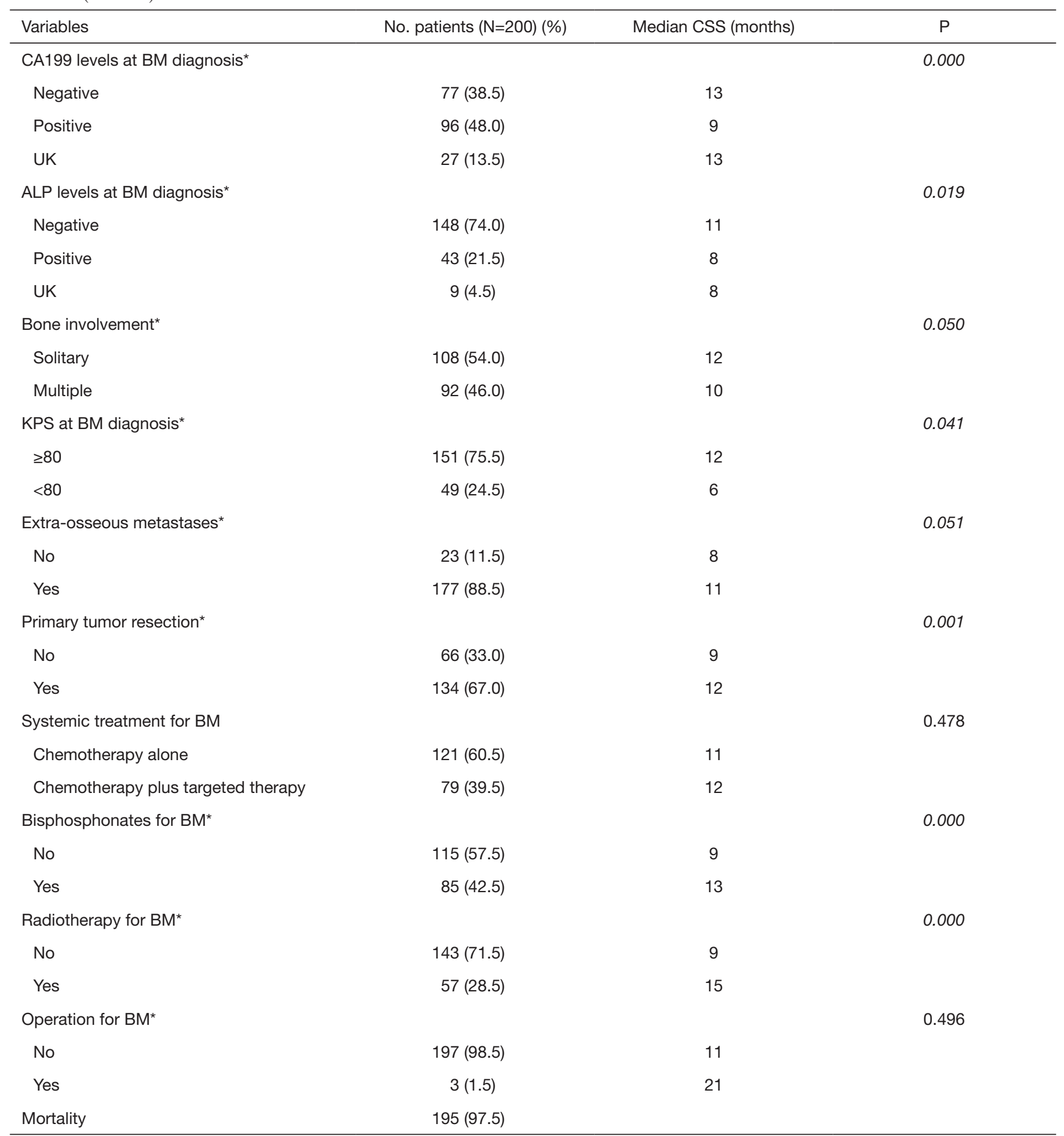

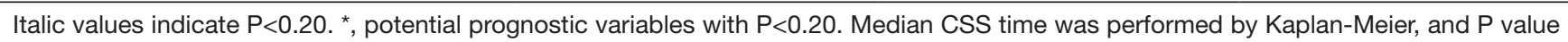
is obtained by Log-Rank test. CSS, cancer specific survival; BM, bone metastasis; N, number; UK, unknown; CEA, carcinoembryonic antigen; CA199, carbohydrate antigen 199; ALP, alkaline phosphatase. 
Table 2 Multivariable COX analysis of prognostic factors for CSS

\begin{tabular}{lcc}
\hline Variables & $\mathrm{HR}(95 \% \mathrm{Cl})$ & $\mathrm{P}$ \\
\hline Basic disease (yes vs. no) & $\mathrm{NA}$ & 0.328 \\
AJCC T stage (T3/T4 vs. T1/T2) & $\mathrm{NA}$ & 0.719 \\
AJCC N stage (N1/N2 vs. N0) & $1.652(1.055-2.587)$ & 0.028 \\
CEA levels at BM diagnosis (positive vs. negative) & $\mathrm{NA}$ & 0.367 \\
CA199 levels at BM diagnosis (positive vs. negative) & $1.460(1.061-2.009)$ & 0.020 \\
ALP levels at BM diagnosis (positive vs. negative) & $\mathrm{NA}$ & 0.827 \\
Bone involvement (multiple vs. solitary) & $1.534(1.117-2.105)$ & 0.008 \\
KPS at BM diagnosis (<80 vs. $\geq 80)$ & $1.527(1.071-2.176)$ & 0.019 \\
Extra-osseous metastases (yes vs. no) & $\mathrm{NA}$ & 0.101 \\
Primary tumor resection (yes vs. no) & $0.627(0.429-0.916)$ & 0.016 \\
Bisphosphonates for BM (yes vs. no) & $0.581(0.429-0.788)$ & 0.479 \\
Radiotherapy for BM (yes vs. no) & $0.578(0.407-0.822)$ & 0.423 \\
\hline
\end{tabular}

Italic values indicate statistical significance at $\mathrm{P}<0.05$. CSS, cancer specific survival; $\mathrm{Cl}$, confidence interval; HR, hazard ratio; $\beta$, beta coefficients; NA, not available; CEA, carcinoembryonic antigen; CA199, carbohydrate antigen 199; ALP, alkaline phosphatase.

bisphosphonates and no radiotherapy were assigned for 2 points, respectively. For the 150 patients (after excluding 50 patients with unknown AJCC N stage and serum CA199 levels), the risk scores ranged from a minimum of 0 to a maximum of 10. Considering equal risk stratification, four groups were finally divided: low risk ( $0-2$ points), medium risk (3-5 points), medium-high risk (6-8 points) and high risk ( $\geq 9$ points) with $35,16,9$ and 5 months of median CSS, respectively $(\mathrm{P}=0.000)$ (Table 3). Medium risk (HR: 2.919, 95\% CI: 1.307-6.517, $\mathrm{P}=0.009$ ), medium-high risk (HR: 6.382, 95\% CI: 2.875-14.166, $\mathrm{P}=0.000$ ) and high risk (HR: 14.149, 95\% CI: 5.060-39.564, $\mathrm{P}=0.000$ ) patients had worse CSS compared with low risk patients. The 1-, 2- and 3-year CSS rate in low risk group was $81.8 \%, 72.7 \%$ and $36.4 \%$, while in high risk group decreased dramatically with $11.1 \%$, $0.0 \%$ and $0.0 \%$, respectively. The time-dependent receiver operating characteristic (timeROC) curves suggested good discrimination of risk stratification to identify the CSS with 1-, 2- and 3-year AUROC of 0.721, 0.810 and 0.823, respectively (Figure 2). Calibration curves for 1-, 2-, and 3 -year CSS estimates showed good correlation between the CSS estimates of risk stratification and Kaplan-Meier estimates (Figure 3).

\section{Discussion}

Our study retrospectively reviewed the clinicopathological characteristics of 200 patients with BM from CRC, discussing prognostic factors and a novel risk stratification for CSS. Many researches have been reported that spine is the leading site of BM from solid tumors $(8,9)$. And the common sites of BM from CRC are reportedly the spine, followed by the pelvis and long bones, which is highly consistent with our study (10).

The prognosis of patients with BM from CRC is very poor because of advanced disease stage, with a median survival time of 7.0 to 17.8 months after BM diagnosis $(6,10,11)$. In our study, the overall median CSS time was 11 months (95\% CI: 9.7-12.3) from the time of BM diagnosis, with $30.0 \%, 17.0 \%$ and $7.0 \%$ of 1-, 2- and 3-year CSS rate. Although the median CSS of patients with adenocarcinoma was longer than those with mucinous adenocarcinoma, signet-ring cell carcinoma and other pathological types (11, 9,8 , and 4 months, respectively), there was no statistical significance $(\mathrm{P}=0.453)$. Median CSS time of patients with metachronous $\mathrm{BM}$ and synchronous $\mathrm{BM}$ were both 11 months. The CSS did not differ significantly between the two groups $(\mathrm{P}=0.571)$ in univariate analysis. Kawamura et al. also found there is no significant difference in survival between metachronous and synchronous groups after BM diagnosis $(\mathrm{P}=0.59)(8)$.

Then we analyzed the prognostic factors based on multivariable analysis, which revealed the CSS in patients with regional lymph node metastasis was found to be shorter 
A

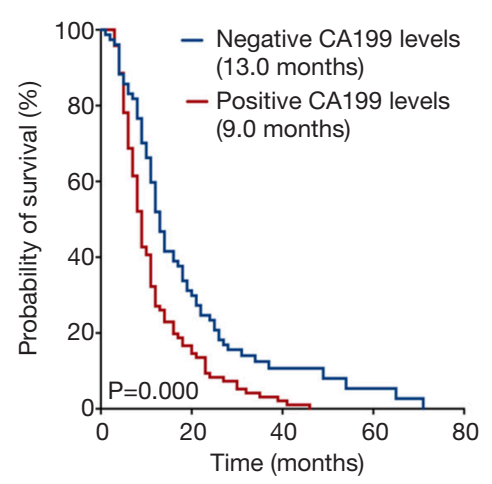

D
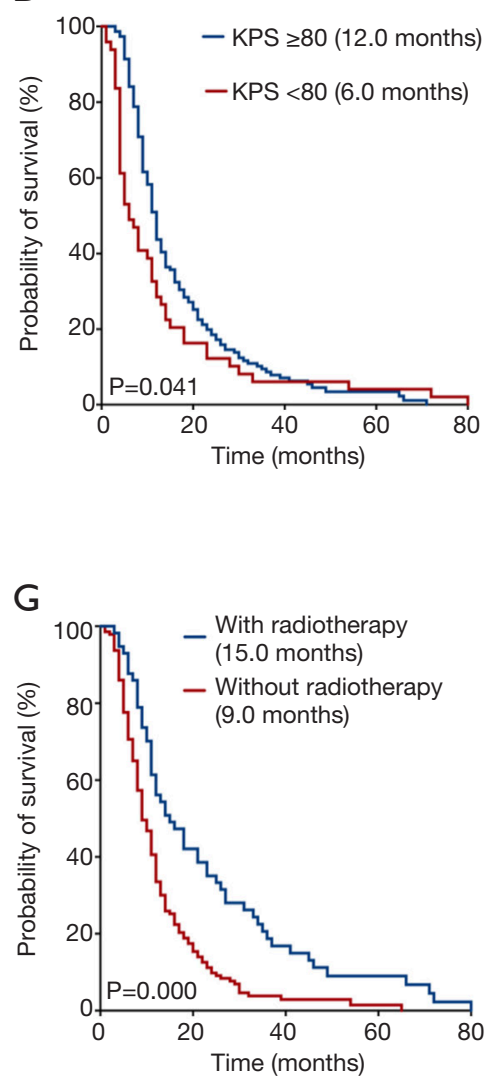

B

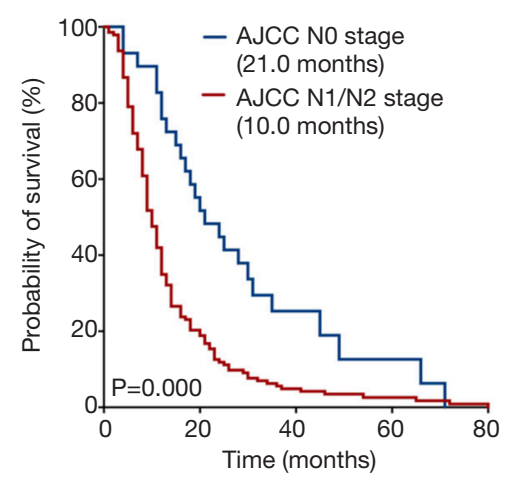

E

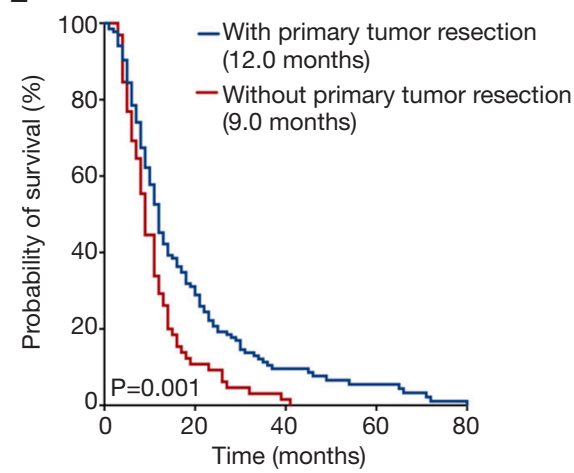

C

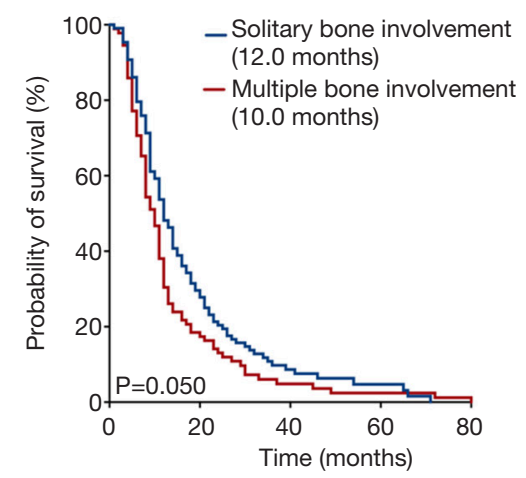

$\mathrm{F}$

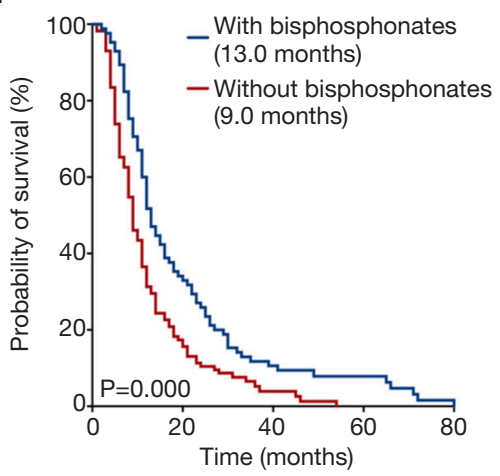

Figure 1 Kaplan-Meier curves of cancer specific survival according to different prognostic variables with $\mathrm{P}<0.05$. (A) Serum CA199 levels at BM diagnosis; (B) AJCC N stage; (C) bone involvement; (D) KPS at BM diagnosis; (E) primary tumor resection; (F) bisphosphonates therapy; (G) radiotherapy. KPS, Karnofsky Performance Status; BM, bone metastasis.

than patients who were without. In addition, patients with positive serum CA199 levels at BM diagnosis had poorer prognosis in CRC patients than those with negative. High CA199 levels is one of indicators of colorectal tumor, but there are rarely reports about the its role in prognosis of
CRC, while most of researches show it plays a key role in CRC diagnosis $(12,13)$. We also found high serum CEA levels and LHD levels were significant prognostic indicators for CSS by univariate analysis, which had been shown in many reports $(10,14)$. So, we suggest careful surveillance in 
Table 3 CSS of four risk groups based on weighted scoring system

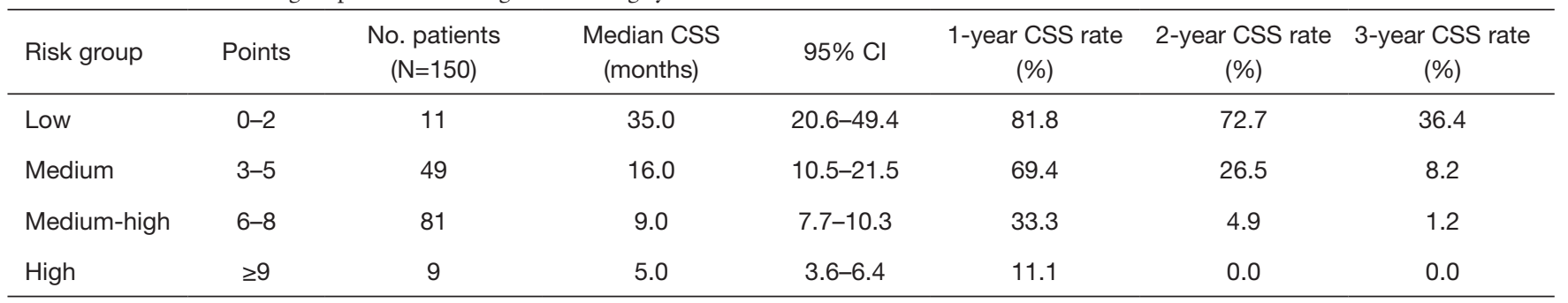

CSS, cancer specific survival; $\mathrm{N}$, number; $\mathrm{Cl}$, confidence interval.

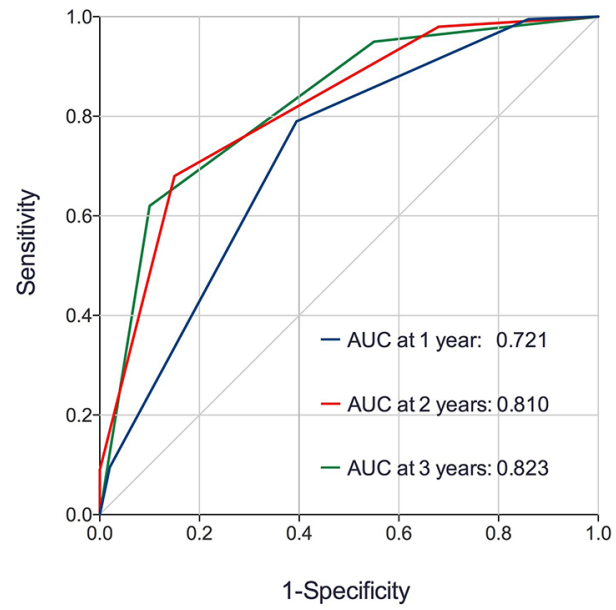

Figure 2 Time-dependent ROC curve for risk stratification. ROC, receiver operating characteristic.

those indicators for patients with BM from CRC.

The multiple bone involvement was found to be an independent prognostic factor for CSS, similar to some previous studies $(15,16)$. However, Lun et al. reported there was no association between the number of bone metastases and survival (17). Such differences might due to the sample size and selection bias in different studies. In our study, KPS scores at BM diagnosis less than 80 were associated with a worse prognosis compared to scores ranging from 80 to 100 . This is in line with previous researches that have identified performance status as one of the most valuable prognostic factors for survival of BM patients (16-18).

There exists controversy regarding to the benefit of primary tumor resection in advanced CRC. Many prior studies suggest a clinical benefit to improve survival with surgery (19-21), while others report there is no benefit $(22,23)$. We found the patients with BM from CRC could
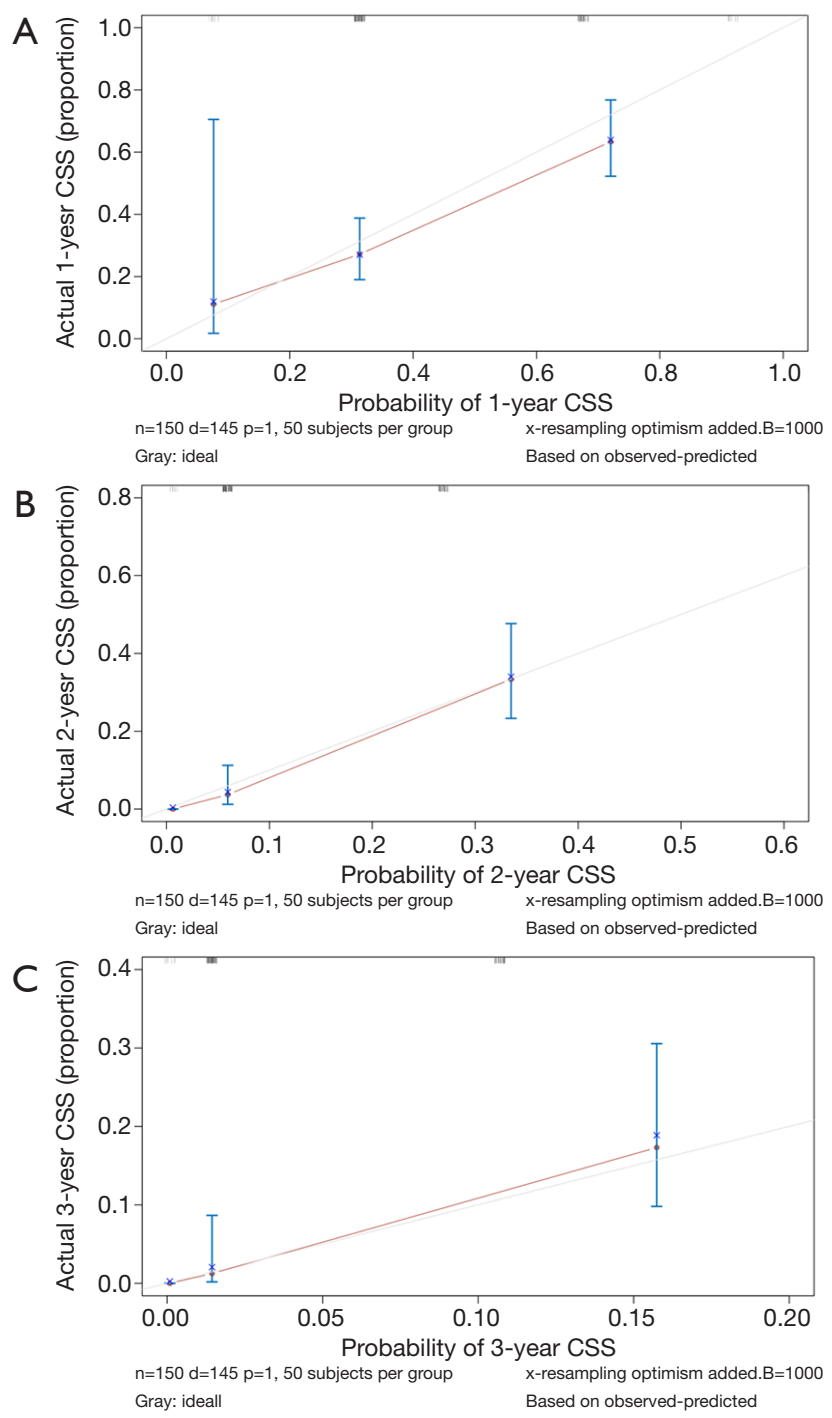

Figure 3 Calibration curves of actual CSS with 95\% CI by decile (y-axis), over predicted CSS (x-axis) by risk stratification: (A) 1-year CSS calibration curve; (B) 2-year calibration curve; (C) 3-year calibration curve. CSS, CSS, cancer specific survival. 
significantly be beneficial from primary tumor resection with improved CSS. That might be because removal of primary tumor could prevent tumor-related complications such as bleeding, obstruction and perforation, further improving quality and survival of patients with BM.

The treatment of bisphosphonates and radiotherapy for CRC patients with BM is strongly recommended. Bisphosphonates have been used for prevention of skeletalrelated events and reduction of pain from $\mathrm{BM}$ in recent years $(24,25)$. According to our study, $42.5 \%$ of patients received bisphosphonates therapy for $\mathrm{BM}$ and the remaining patients didn't. The difference in median CSS between two groups is significant (13vs. 9 months, respectively), showing that patients who took bisphosphonates were associated with better prognosis.

Radiotherapy was found to be associated with longer CSS, although quality-of-life data were lacking. In patients with palliative radiotherapy for BM, median CSS time after BM diagnosis was prolonged obviously (15 vs. 9 months, respectively). Previous researches have verified that radiotherapy is the approach most commonly used to treat severe pain from BM, which could improve survival directly and indirectly (26-28). In addition, the chemotherapy can notably improve survival of BM as many researches have demonstrated $(26,29,30)$. However, because all patients had received adjuvant chemotherapy in our study, the utility of chemotherapy in improving CSS was not investigated.

Here, we developed a weighted scoring system to facilitate risk stratification for patients with BM from CRC. As an example, a CRC patient, with regional lymph node metastasis, negative CA199 levels, KPS $\geq 80$ and multiple bone involvement, received primary tumor resection, bisphosphonates therapy and radiotherapy for BM, then he would be assigned for 0 point with a median CSS benefit of up to 35 months (95\% CI: 20.6-49.4). In contrast, patients with all adverse factors (10 points) showed the worst CSS of only 5 months (95\% CI: 3.6-6.4). So, we suggest that more medical care might be necessary for high risk patients. And individualized medical care should be considered for patients in different risk groups. This risk stratification had good discrimination and calibration, implying a clinical value in predicting prognosis of CRC patients with $\mathrm{BM}$.

Our study had some limitations. First, this was a retrospective designed study, and some data might have missed. For example, not every patient showed complete AJCC TNM stage. In addition, this was a single-center study. Because of the low incidence of BM from CRC, the number of patients was small, which limited the external validation. Finally, because the treatment process for CRC patients with BM is very complicated, many potential prognostic factors including lymphocyte count, inflammation, nutritional status and sarcopenia are missing to a large extent. Therefore, it is regretful that we could not accurately and objectively evaluate the impact of these factors on the prognosis. Nonetheless, we believe our study will be useful to both clinicians and patients.

\section{Conclusions}

Given the poor survival of BM from CRC, information regarding how to evaluate the prognosis of such patients should be considered in diagnosis and treatment. Here, we found AJCC N stage, CA199 levels, multiple bone involvement, KPS scores, primary tumor resection, bisphosphonates and radiotherapy were prognostic factors significantly affecting survival. Four risk groups showed significant differences in CSS, which could help physicians determine the prognosis of CRC patients with BM. The novel risk stratification has considerable practical implications and may be useful in selection of appreciate care and treatment.

\section{Acknowledgments}

Funding: This work was supported by Sanming Project of Medicine in Shenzhen (No. SZSM201911012).

\section{Footnote}

Reporting Checklist: The authors have completed the Tripod reporting checklist. Available at http://dx.doi.org/10.21037/ jgo-20-586

Data Sharing Statement: Available at http://dx.doi. org/10.21037/jgo-20-586

Peer Review File: Available at http://dx.doi.org/10.21037/ jgo-20-586

Conflicts of Interest: All authors have completed the ICMJE uniform disclosure form (available at http://dx.doi. org/10.21037/jgo-20-586). The authors have no conflicts of interest to declare.

Ethical Statement: The authors are accountable for all aspects of the work in ensuring that questions related 
to the accuracy or integrity of any part of the work are appropriately investigated and resolved. The study was conducted in accordance with the Declaration of Helsinki (as revised in 2013). This study was approved by the Ethics Committee of Cancer Hospital, Chinese Academy of Medical Sciences (NCC2019S-060) and informed consent was taken from all the patients.

Open Access Statement: This is an Open Access article distributed in accordance with the Creative Commons Attribution-NonCommercial-NoDerivs 4.0 International License (CC BY-NC-ND 4.0), which permits the noncommercial replication and distribution of the article with the strict proviso that no changes or edits are made and the original work is properly cited (including links to both the formal publication through the relevant DOI and the license). See: https://creativecommons.org/licenses/by-nc-nd/4.0/.

\section{References}

1. Bray F, Ferlay J, Soerjomataram I, et al. Global Cancer Statistics 2018: GLOBOCAN Estimates of Incidence and Mortality Worldwide for 36 Cancers in 185 Countries. CA Cancer J Clin 2018;68:394-424.

2. Disibio G, French SW. Metastatic patterns of cancers: results from a large autopsy study. Arch Pathol Lab Med 2008;132:931-9.

3. Kanthan R, Loewy J, Kanthan S. Skeletal metastases in colorectal carcinomas: a Saskatchewan profile. Dis Colon Rectum 1999;42:1592-7.

4. Sundermeyer ML, Meropol NJ, Rogatko A, et al. Changing patterns of bone and brain metastases in patients with colorectal cancer. Clin Colorectal Cancer 2005;5:108-13.

5. Nozue M, Oshiro Y, Kurata M, et al. Treatment and prognosis in colorectal cancer patients with bone metastasis. Oncol Rep 2002;9:109-12.

6. Baek SJ, Hur H, Min BS, et al. The Characteristics of Bone Metastasis in Patients with Colorectal Cancer: A Long-Term Report from a Single Institution. World J Surg 2016;40:982-6.

7. Kang SJ, Cho YR, Park GM, et al. Predictors for functionally significant in-stent restenosis: an integrated analysis using coronary angiography, IVUS, and myocardial perfusion imaging. JACC Cardiovasc Imaging 2013;6:1183-90.

8. Kawamura H, Yamaguchi T, Yano Y, et al. Characteristics and Prognostic Factors of Bone
Metastasis in Patients With Colorectal Cancer. Dis Colon Rectum 2018;61:673-8.

9. Roodman GD. Mechanisms of bone metastasis. N Engl J Med 2004;350:1655-64.

10. Wolmark N, Fisher B, Wieand HS, et al. The prognostic significance of preoperative carcinoembryonic antigen levels in colorectal cancer. Results from NSABP (National Surgical Adjuvant Breast and Bowel Project) clinical trials. Ann Surg 1984;199:375-82.

11. Liu F, Zhao J, Xie J, et al. Prognostic risk factors in patients with bone metastasis from colorectal cancer. Tumour Biol 2016. [Epub ahead of print]. doi: 10.1007/ s13277-016-5465-4.

12. Yang $\mathrm{W}$, Luo $\mathrm{Y}, \mathrm{Hu} \mathrm{S}$, et al. Value of combined detection of serum carcino-embryonic antigen, carbohydrate antigen 19-9 and cyclooxygenase-2 in the diagnosis of colorectal cancer. Oncol Lett 2018;16:1551-6.

13. Zhong W, Yu Z, Zhan J, et al. Association of Serum Levels of CEA, CA199, CA125, CYFRA21-1 and CA72-4 and Disease Characteristics in Colorectal Cancer. Pathol Oncol Res 2015;21:83-95.

14. Ulas A, Bilici A, Durnali A, et al. Risk factors for skeletalrelated events (SREs) and factors affecting SRE-free survival for nonsmall cell lung cancer patients with bone metastases. Tumour Biol 2016;37:1131-40.

15. Choi D, Fox Z, Albert T, et al. Prediction of Quality of Life and Survival After Surgery for Symptomatic Spinal Metastases: A Multicenter Cohort Study to Determine Suitability for Surgical Treatment. Neurosurgery 2015;77:698-708.

16. Park HS, Rha SY, Kim HS, et al. A Prognostic Model to Predict Clinical Outcome in Gastric Cancer Patients with Bone Metastasis. Oncology 2011;80:142-50.

17. Lun DX, Xu LN, Wang F,et al. Prognostic Differences in Patients with Solitary and Multiple Spinal Metastases. Orthop Surg 2019;11:443-50.

18. Tabouret E, Gravis G, Cauvin C, et al. Long-term survivors after surgical management of metastatic spinal cord compression. Eur Spine J 2015;24:209-15.

19. Park JH, Kim TY, Lee KH, et al. The beneficial effect of palliative resection in metastatic colorectal cancer. $\mathrm{Br} \mathrm{J}$ Cancer 2013;108:1425-31.

20. Gulack BC, Nussbaum DP, Keenan JE, et al. Surgical Resection of the Primary Tumor in Stage IV Colorectal Cancer Without Metastasectomy Is Associated With Improved Overall Survival Compared With Chemotherapy/Radiation Therapy Alone. Dis Colon Rectum 2016;59:299. 
21. Maroney S, de Paz CC, Reeves ME, et al. Benefit of Surgical Resection of the Primary Tumor in Patients Undergoing Chemotherapy for Stage IV Colorectal Cancer with Unresected Metastasis. J Gastrointest Surg 2018;22:460-6.

22. Matsumoto T, Hasegawa S, Matsumoto S,et al. Overcoming the Challenges of Primary Tumor Management in Patients With Metastatic Colorectal Cancer Unresectable for Cure and an Asymptomatic Primary Tumor. Dis Colon Rectum 2014;57:679-86.

23. Massarweh NN, Li LT, Sansgiry S, et al. Primary Tumor Resection and Multimodality Treatment for Patients with Metastatic Colon Cancer. Ann Surg Oncol 2016;23:1815-23.

24. Drake MT, Clarke BL, Khosla S. Bisphosphonates: Mechanism of Action and Role in Clinical Practice. Mayo Clin Proc 2008;83:1032-45.

25. Rennert G, Pinchev M, Rennert HS, et al. Use of Bisphosphonates and Reduced Risk of Colorectal Cancer. J Clin Oncol 2011;29:1146-50.

Cite this article as: Ma X, Guan X, Ma C, Quan J, Zhao Z, Chen H, Huang H, Wei R, Liu Z, Jiang Z, Chen Y, Wang X. A novel risk stratification for predicting prognosis of colorectal cancer patients with bone metastasis. J Gastrointest Oncol 2021;12(3):933-943. doi: 10.21037/jgo-20-586
26. Valentini V, van Stiphout RG, Lammering G, et al. Nomograms for predicting local recurrence, distant metastases, and overall survival for patients with locally advanced rectal cancer on the basis of European randomized clinical trials. J Clin Oncol 2011;29:3163-72.

27. Chow E, Harris K, Fan G, et al. Palliative radiotherapy trials for bone metastases: a systematic review. J Clin Oncol 2007;25:1423.

28. McDonald R, Ding K, Brundage M, et al. Effect of Radiotherapy on Painful Bone Metastases: A Secondary Analysis of the NCIC Clinical Trials Group Symptom Control Trial SC.23. JAMA Oncol 2017;3:953-9.

29. Van Cutsem E, Cervantes A, Adam R, et al. ESMO consensus guidelines for the management of patients with metastatic colorectal cancer. Ann Oncol 2016;27:1386-422.

30. Kobayashi H, Kotake K, Sugihara K. Impact of adjuvant chemotherapy in patients with curatively resected stage IV colorectal cancer. Medicine 2015;94:e696. 